OPEN ACCESS

Edited by:

Shijie Sheng,

Wayne State University, United States

Reviewed by:

Natasa Djordjevic,

University of Kragujevac, Serbia

Lingfei Wang,

Baylor College of Medicine,

United States

*Correspondence:

Rodolfo Montironi

r.montironi@univpm.it

Francesco Piva

f.piva@staff.univpm.it

Specialty section:

This article was submitted to

Genomic Medicine,

a section of the journal

Frontiers in Genetics

Received: 27 September 2019

Accepted: 23 March 2020

Published: 15 April 2020

Citation:

Occhipinti G, Romagnoli E,

Santoni M, Cimadamore A, Sorgentoni G, Cecati M, Giulietti M, Battelli N, Maccioni A, Storti N,

Cheng L, Principato G, Montironi $R$ and Piva $F$ (2020) Sequential or

Concomitant Inhibition

of Cyclin-Dependent Kinase 4/6

Before mTOR Pathway

in Hormone-Positive HER2 Negative Breast Cancer: Biological Insights

and Clinical Implications.

Front. Genet. 11:349.

doi: 10.3389/fgene.2020.00349

\section{Sequential or Concomitant Inhibition of Cyclin-Dependent Kinase 4/6 Before mTOR Pathway in Hormone-Positive HER2 Negative Breast Cancer: Biological Insights and Clinical Implications}

Giulia Occhipinti ${ }^{1}$, Emanuela Romagnoli ${ }^{2}$, Matteo Santoni ${ }^{2}$, Alessia Cimadamore ${ }^{3}$, Giulia Sorgentoni ${ }^{2}$, Monia Cecati ${ }^{1}$, Matteo Giulietti ${ }^{1}$, Nicola Battelli ${ }^{2}$, Alessandro Maccioni ${ }^{4}$, Nadia Storti5, Liang Cheng', Giovanni Principato', Rodolfo Montironi ${ }^{3 *}$ and Francesco Piva ${ }^{1 *}$

\footnotetext{
Department of Specialistic Clinical and Odontostomatological Sciences, Polytechnic University of Marche, Ancona, Italy, ${ }^{2}$ Oncology Unit, Macerata Hospital, Macerata, Italy, ${ }^{3}$ Section of Pathological Anatomy, School of Medicine, United Hospitals, Polytechnic University of the Marche Region, Ancona, Italy, ${ }^{4}$ Direzione Area Vasta 3, Macerata, Italy, ${ }^{5}$ Direzione Sanitaria Azienda Sanitaria Unica Regionale, Ancona, Italy, ${ }^{6}$ Department of Pathology and Laboratory Medicine, Indiana University School of Medicine, Indianapolis, IN, United States
}

About $75 \%$ of all breast cancers are hormone receptor-positive (HR+). However, the efficacy of endocrine therapy is limited due to the high rate of either pre-existing or acquired resistance. In this work we reconstructed the pathways around estrogen receptor (ER), mTOR, and cyclin D in order to compare the effects of CDK4/6 and PI3K/AKT/mTOR inhibitors. A positive feedback loop links mTOR and ER that support each other. We subsequently considered whether a combined or sequential inhibition of CDK4/6 and PI3K/AKT/mTOR could ensure better results. Studies indicate that inhibition of CDK4/6 activates mTOR as an escape mechanism to ensure cell proliferation. In literature, the little evidence dealing with this topic suggests that pretreatment with mTOR pathway inhibitors could prevent or delay the onset of CDK4/6 inhibitor resistance. Additional studies are needed in order to find biomarkers that can identify patients who will develop this resistance and in whom the sensitivity to CDK4/6 inhibitors can be restored.

Keywords: breast cancer, estrogen receptor, cyclin-dependent kinases (CDKs), CDK inhibitors, mechanisms of resistance

\section{INTRODUCTION}

Breast cancer presents a significant health burden worldwide according to the International Agency for Research on Cancer ${ }^{1}$. About $75 \%$ of all breast cancers are hormone receptor-positive $(\mathrm{HR}+) . \mathrm{HR}+$ cancers express estrogen receptors (ER) and/or progesterone receptors (PgR). Such tumors are basically dependent on the ER-related signaling pathway in order to grow and survive.

${ }^{1}$ http://globocan.iarc.fr/Pages/fact_sheets_cancer.aspx 
The ER-related signaling pathway regulates various cellular functions, such as apoptosis and cell proliferation as well as angiogenesis. $\mathrm{HR}+$ cancers take advantages of the ER pathway in order to promote cancer development, growth, and progression (Platet et al., 2004). This has led to the development of therapeutic agents targeting the estrogen signaling pathway, i.e., aromatase inhibitors (AIs; such as exemestane, anastrozole and letrozole), selective down-regulators of ERs (fulvestrant), selective modulators of ERs (tamoxifen). This endocrine therapy has become the treatment of choice for breast cancers that are HR+ (Milla-Santos et al., 2003; Di Leo et al., 2014).

The efficacy of such a therapeutic approach is however limited. This, due to the high rate of pre-existing as well as acquired resistance (Osborne and Schiff, 2011; De Marchi et al., 2016), has led to a proportion of patients failing to respond to endocrine therapy. Resistance to endocrine therapy is an important problem from the clinical point of view.

\section{Target Therapy: mTOR Inhibitors}

Information on the $\mathrm{PI} 3 \mathrm{~K} / \mathrm{AKT} / \mathrm{mTOR}$ pathway has led to the development of mTOR inhibitors (Hosford and Miller, 2014), including Everolimus. In 2012 this drug was approved for the treatment of ER+/HER2- metastatic cancer following progression on previous non-steroidal AI.

Effectiveness of combining endocrine therapy with mTOR inhibitors has been tested in several trials, including the TAMRAD trial, a randomized phase 2 trial of tamoxifen with or without everolimus in postmenopausal women with AIresistant, ER+, advanced cancer. The combination arm was associated with a significantly better progression-free survival (PFS; 4.5 vs. 8.6 months) and overall survival (OS) (Bachelot et al., 2012). BOLERO-2 is a phase 3 trial of exemestane combined with either everolimus or placebo. This was conducted in postmenopausal women with advanced ER+/HER2- cancer resistant to either letrozole or anastrozole. The study showed an improvement in PFS (3.2 months in the placebo/exemestane arm vs. 7.8 months in the everolimus/exemestane arm) (Yardley et al., 2013). Moreover, everolimus resulted to be effective in combination with fulvestrant in AI-resistant luminal metastatic cancer (NCT01797120) or with letrozole as first-line treatment in postmenopausal patients with luminal metastatic cancer (NCT01698918).

\section{Target Therapy: CDK 4/6 Inhibitors}

The advent of cyclin-dependent kinases (CDKs) 4/6 inhibitorbased combination therapies represents a challenge for breast cancer treatment. The development of inhibitors of CDK4 and CDK6 has changed the perception of CDKs as therapeutic targets in breast cancer following "underwhelming results and unacceptable toxicity were seen with pan-CDK inhibitors such as flavopiridol (alvocidib) in the early 2000s" (Fry et al., 2004; Toogood et al., 2005; Hosford and Miller, 2014; Asghar et al., 2015). Palbociclib is an orally active pyridopyrimidine, highly selective reversible inhibitor of CDK4 and CDK6. The effect of palbociclib is linked on the presence of a functional retinoblastoma protein $(\mathrm{Rb})$, whereas there is no effect on $\mathrm{Rb}$ deficient cells (Kim et al., 2014). On the other hand, abemaciclib, and ribociclib are selective small molecule reversible inhibitors of CDK4/6. It is hypothesized that the selectivity of palbociclib, abemaciclib, and ribociclib is due to the specific interactions with aminoacids of the ATP-binding site in the catalytic cleft of CDK4 and CDK6 (Asghar et al., 2015).

At present, no studies have directly compared the safety and efficacy of the two sequences CDK4/6 inhibitor/everolimus vs. everolimus/CDK4/6 inhibitor. Our computational study aims to show the inhibition of the pathways by the CDK $4 / 6$ inhibitors and everolimus and the biological rational of sequencing or combining these two therapeutic strategies. Literature search has been performed in PubMed by using combinations of specific search terms, such as "breast," "resistance," “CDK4," “CDK6," “mTOR," "PI3K," “inhibitor," or the corresponding drug names and their synonyms. We have selected research articles from 2001 focusing on ER+ or triple negative cancer cell lines, human ER+ breast tumor samples and patient derived xenograft models. Finally, some review articles, analyzing alterations in the $\mathrm{PI} 3 \mathrm{~K} / \mathrm{AKT} / \mathrm{mTOR}$ pathway in ER+ breast tumors associated with endocrine therapy resistance, where selected.

\section{CELL CYCLE SIGNALING PATHWAY}

Cell cycle is regulated by the CDK4/6-Rb-E2F axis. Several other factors strictly control this axis including cyclin D, INK4 family proteins $\left(\mathrm{p} 15^{\mathrm{INK} 4 \mathrm{~b}}, \mathrm{p} 16^{\mathrm{INK} 4 \mathrm{a}}, \mathrm{p} 18^{\mathrm{INK} 4 \mathrm{c}}\right.$, and $\left.\mathrm{p} 19^{\mathrm{INK} 4 \mathrm{~d}}\right), \mathrm{p} 21^{\mathrm{CIP} 1}$, and p27 ${ }^{\text {Kip1 }}$ (Olmez et al., 2017).

The activated cyclin D-CDK4/6 complex provokes full phosphorylation and functional inactivation of $\mathrm{Rb}$. $\mathrm{Rb}$ active form consists in its hypo-phosphorylated status. In this way, active $\mathrm{Rb}$ acts as tumor suppressor primarily through binding and consequent suppression of the nuclear E2F family of transcription factors as well as by governing p27Kip1 stability partly through interacting with $\mathrm{APC}^{C d h 1}$; this causes cell cycle arrest in the transition from G1 to $S$ phase. Hyperphosphorylated $\mathrm{Rb}$ loses its suppressive role toward $\mathrm{E} 2 \mathrm{~F}$ in the nucleus leading to the expression of E2F target genes required for S-phase entry. In the cytoplasm, hyper-phosphorylated $\mathrm{Rb}$ binds Sin1, a component of mTORC2 complex, thus preventing AKT phosphorylation and mTORC1 activation (Zhang et al., 2016; Bonelli et al., 2017; Figure 1). mTOR signaling is activated by a variety of different signals such as EGF, IGF-1, amino acids or high cellular energy. Growth factors activate the phosphatidylinositol 3-kinase (PI3K) that phosphorylates PIP2 to PIP3. The increased amount of PIP3 allows the co-localization of the serine/threonine kinase AKT, phosphoinositide-dependent kinase-1 (PDK1) and mTORC2 to the plasma membrane. PDK1 and mTORC2 phosphorylate AKT leading to the complete AKT activation that, in turn, activates mTORC1 (Kim et al., 2017). mTORC1 complex (i) activates the transcription factor $\mathrm{HIF} 1 \alpha$ that induces cell growth and angiogenesis, (ii) releases the translation factor eIF4E that enhances mRNA expression and export of genes as cyclin $\mathrm{D}$, therefore favoring cell proliferation (Rosenwald et al., 1995; Michaloglou et al., 2018), (iii) activates the $40 \mathrm{~S}$ 


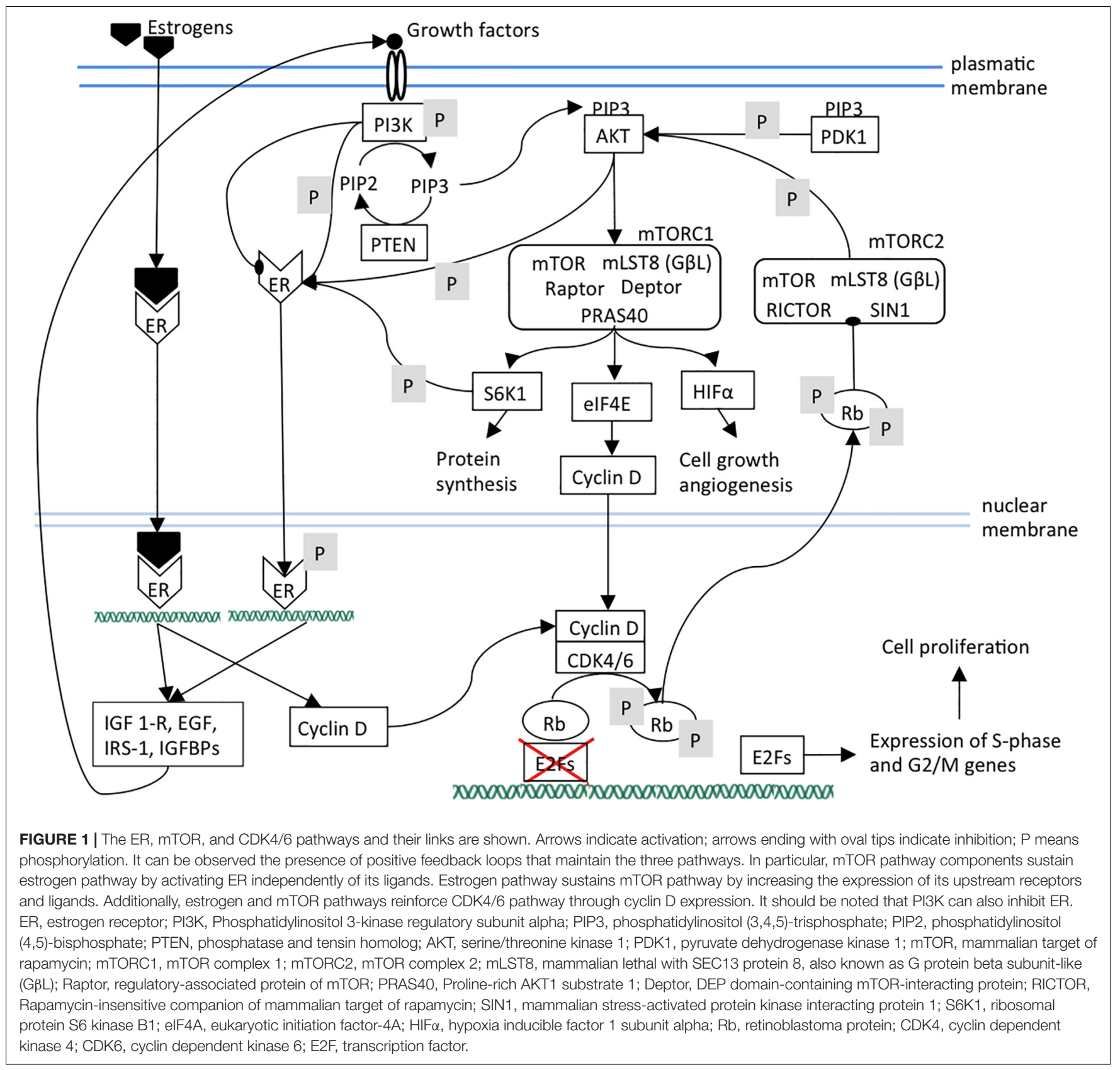

ribosomal S6 kinase 1 (S6K1) that increases protein synthesis (Michaloglou et al., 2018).

Briefly, $\mathrm{Rb}$ is involved in two independent pathways. Hypophosphorylated $\mathrm{Rb}$ suppresses $\mathrm{E} 2 \mathrm{~F} 1$, inducing the cell cycle arrest at G1 phase. The hyper-phosphorylated Rb stops the AKT oncogenic signaling pathways through mTORC2 inhibition (Zhang et al., 2016).

Estrogens stimulate cell proliferation by increasing cyclin $\mathrm{D}$ expression, but also in an indirect way through mTOR. In fact, PI3K/AKT/mTOR cascade is also involved in ER pathway triggering since S6K1, AKT, and PI3K induce ER phosphorylation and activation independently to the estrogen presence (Campbell et al., 2001; Yamnik et al., 2009). Consequently, despite the estrogen deprivation, ER signaling can be activated resulting in endocrine therapy resistance. The trigger of the ER signaling pathway, both estrogen-dependent and independent, induces the transcription of genes (such as IGF1-R, EGF, IRS-1, IGFBPs) encoding for $\mathrm{PI} 3 \mathrm{~K} / \mathrm{AKT} / \mathrm{mTOR}$ pathway effectors resulting in a positive feedback loop (Miller et al., 2011; Ciruelos Gil, 2014; Figure 1). However, the interaction between these two pathways seems more complicated, since there could be even an inverse correlation of PI3K activation with ER expression levels. In a model of ER+ cancer, PI3K inhibition led to an increase of ER expression, whereas PI3K activation induced a decrease of ER expression level (Creighton et al., 2010). This evidence is supported by another study which demonstrated that in 
ER + breast cancer models the PI3K inhibition enhanced the expression of ER at both mRNA and at protein levels. This, in turn, promoted the expression of the genes regulated by ER (Bosch et al., 2015).

The combination of mTOR or PI3K inhibitors to CDK4/6 inhibitor therapy have shown encouraging preliminary results in clinical trials, although PI3K inhibitors resulted to be quite toxic and poorly effective and, therefore, they are not yet approved for breast cancer (Hamilton and Infante, 2016; Cortes et al., 2017; Ballinger et al., 2018).

\section{Sequential Inhibition of CDK4/6 and mTOR}

The blockade of CDK4/6 activity by therapeutic inhibitors leads to $\mathrm{Rb}$ de-phosphorylation and to the subsequent strengthening of $\mathrm{PI} 3 \mathrm{~K} / \mathrm{AKT} / \mathrm{mTOR}$ pathway that plays a pivotal role in the control of cell growth, migration and metabolism (Cretella et al., 2018; Figure 2). mTORC2 inhibition lowers cell proliferation by weakening of mTORC1, cyclin D, Rb axis. Additionally, ligandindependent ER phosphorylation is reduced, leading to a lower expression of growth factors and receptors and therefore to a lower mTOR activation (Figure 3).

Recently, it has been showed that treatment with palbociclib alone for $24 \mathrm{~h}$ in TNBC (triple negative breast cancer) cell lines increases AKT activation. After palbociclib preincubation, the sequential combined treatment with PI3K inhibitors plus palbociclib was associated with G0/G1 cell cycle arrest in a greater percentage of cells. This sequential combined treatment determined a higher mTOR inhibition and $\mathrm{Rb}$ de-phosphorylation, in comparison with the single or simultaneous drug treatment. Notably, the sequential treatment induced a stronger impairment of the glucose metabolism. In fact, palbociclib treatment maintains $\mathrm{Rb}$ in its active form with the consequent repression of E2F transcription factor and downregulation of the transcription factor c-myc, a direct target of E2F. In turn, c-myc down-regulation results in decreased expression of the GLUT-1 glucose transporter. The combination of palbociclib with BYL719 (PI3K inhibitor) enhanced these effects under normoxic and hypoxic conditions, since the inhibition of PI3K/mTOR signaling is also involved in glucose uptake decrease (Cretella et al., 2018).

The synergistic effect on cell cycle arrest and senescence of sequential treatment with palbociclib and PI3K/mTOR inhibitors was observed also in malignant pleural mesothelioma cell models (Bonelli et al., 2017).

\section{Combined Inhibition of CDK4/6 and mTOR}

ER + breast cancer frequently shows a hyperactivation of the mTOR pathway. Some studies have shown advantage from combining mTOR inhibitors with estrogen receptor blockade (Bachelot et al., 2012; Yardley et al., 2013). However, the precise relationship among ER signaling and mTOR pathways has not been clarified. Moreover, activation of CDK-Rb-E2F signaling has often been associated with endocrine resistance; for this reason, CDK4/6 inhibitors, such as palbociclib, are investigated in clinical trials in ER+ cancer. In two breast cancer cell lines, MCF-7 and ER+HCC-1428, the mTORC1/2 inhibitor vistusertib (AZD2014) caused decreased levels of cyclin D1 resulting in the hypo-phosphorylation of $\mathrm{Rb}$, which in turn modulates E2F mediated transcription. However, the combination with palbociclib did not cause a complete growth inhibition and an enhanced senescence-like phenotype, instead it resulted in a prolonged quiescent state (Michaloglou et al., 2018).

In addition, a preclinical study assessing the effects of palbociclib and the mTOR inhibitor sapanisertib, showed that their combination synergistically inhibited breast cancer cell proliferation (Yamamoto et al., 2019).

\section{IMPLICATION FOR TREATMENTS IN BREAST CANCER}

The selection of systemic therapy in patients with advanced cancers is mainly related to HR and HER2 status, previous systemic therapies, and patient status. ER activity is effectively inhibited by endocrine agents. In addition, estrogen/ER complexes can also interact with AP-1 and SP-1 transcription factors which are able to modulate different transcriptional programs (Milla-Santos et al., 2003). On the other hand, the non-nuclear mechanism of action of ER, which can be bound to plasma membrane or free in the cytoplasm, consists in the activation of RTKs (Receptor Tyrosine Kinases) family receptors and, in turn, RTKs trigger their downstream pathways, such as PI3K/AKT/mTOR and Ras/MAPK (Giuliano et al., 2013).

The approval of modern CDK inhibitors has changed the treatment paradigm for advanced $\mathrm{HR}+$ cancer. The use of palbociclib, abemaciclib and ribociclib, that are selective reversible inhibitors of CDK4 and CDK6, has been approved based on progression free survival benefit seen on phase III studies (Laderian and Fojo, 2017). Except for ER positivity, no other biomarkers predictive of response to CDK4/6 inhibitors have been identified so far.

\section{Molecular Mechanism of Resistance to CDK4/6 Inhibitors}

Different molecular mechanisms of resistance to CDK4/6 inhibitors can arise (Pandey et al., 2019). They are due to p16, CDK6, CCNE1/2, CDK2, CDK4, or E2F amplification and also to loss of or mutations in $\mathrm{Rb}$. It is remarkable that the loss of $\mathrm{Rb}$ is responsible for activation of E2F and the cyclin E-CDK2 axis, assuring progression of the cell cycle. Recently, it was elucidated that also loss of the FAT1 (FAT atypical cadherin 1) tumor suppressor can promote resistance to CDK4/6 inhibitors (Li et al., 2018). Therefore, there are different mechanisms of early resistance to CDK4/6 inhibitors that favor alternative pathways of S-phase entry.

CDK4/6 inhibitors or CDK4/6 inhibitor-resistant breast cancer cells activate the PI3K/AKT/mTOR pathway (HerreraAbreu et al., 2016; Jansen et al., 2017; Pandey et al., 2019). mTOR promotes cell proliferation and growth by suppressing autophagy and promoting anabolic processes, such as biosynthesis of lipids and proteins. mTOR could have a more complex role since 


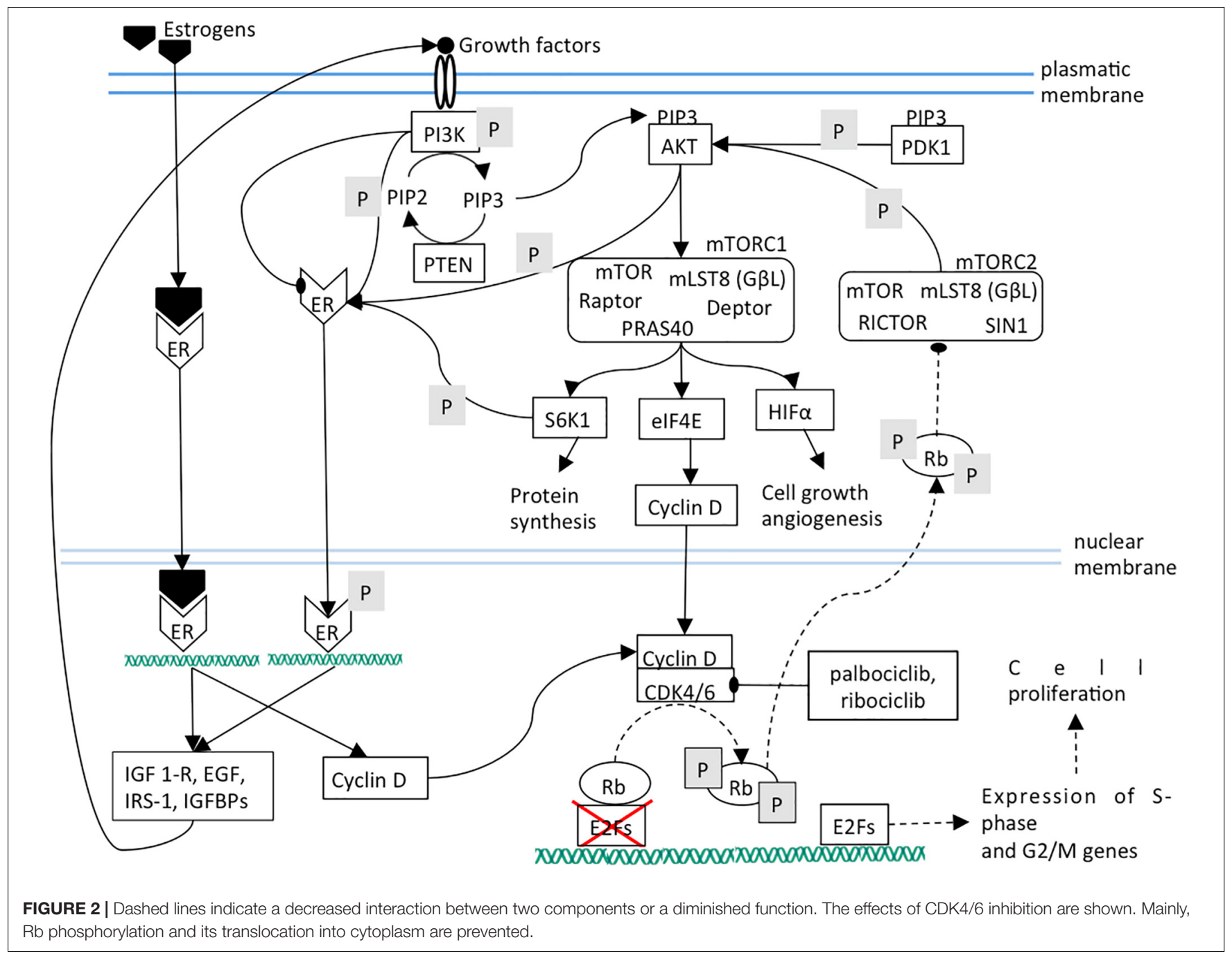

it also localizes in nucleus where it interacts with epigenetic regulators. A recent study by Michaloglou et al. (2018) suggests that, in CDK4/6 inhibitor-resistant ER-positive cancer cell lines, inhibition of mTORC1/2 restores sensitivity to CDK $4 / 6$ inhibitors (Michaloglou et al., 2018). Another investigation shows that PI3K inhibitors are able to prevent the resistance to CDK4/6 inhibitors. However, it fails to re-sensitize cells when resistance is acquired (Herrera-Abreu et al., 2016). According to the above mentioned in vitro studies, it seems better to hit mTOR first in order to avoid or delay tolerance to CDK4/6 inhibitors. Accordingly, in a small clinical trial including 23 metastatic HR+/HER2- breast cancer patients treated with palbociclib after pretreatment with everolimus, it has been reported a limited clinical benefit, since poor response rate and short PFS have been observed (Dhakal et al., 2018). Nevertheless, this data should be confirmed in randomized clinical trials comparing the sequences mTOR-CDK4/6 inhibitors vs. CDK4/6-mTOR inhibitors in patients with breast cancer.

It is important to note that this pathway reconstruction could have various limitations, including missing actors, unknown interactions and particular cases, such as gene amplifications or mutations that could cause the loss of function of some elements not taken into account.

\section{CDK4/6 Inhibitors in Combination With Other Therapies}

The use of CDK4/6 inhibitors is being investigated in combination with other drugs, i.e., targeted therapy, immunotherapy and chemotherapy.

Pre-clinical data suggest that there is a synergistic effect when agents targeting the PI3K/mTOR pathway are combined with CDK4/6 inhibition. Preliminary information on a few patients has been observed when mTOR inhibitors (everolimus) and $\alpha$-specific PI3K inhibitor (alpelisib) are used with abemaciclib and ribociclib. A phase Ib trial of ribociclib, everolimus, and exemestane in 83 pre-treated patients with HR+/HER2advanced cancer showed an Overall Response Rate (ORR) of $13 \%$. Twenty-three percent of the patients had received prior PI3K/AKT/mTOR inhibitors (Oliveira et al., 2016). The combined therapy was basically well tolerated and the safety profile was consistent with the combination of everolimus 


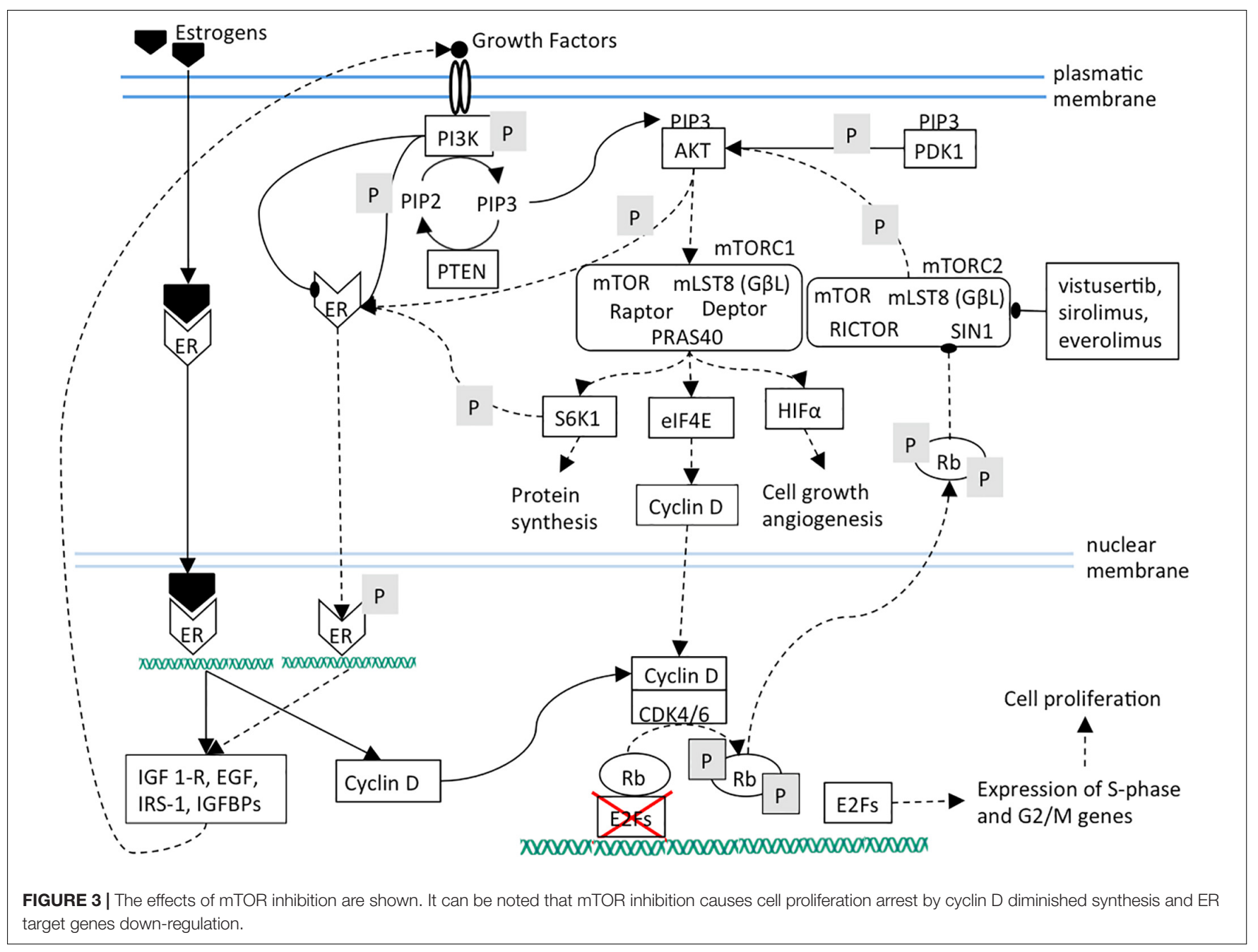

and exemestane. Furthermore, abemaciclib was investigated in combination with everolimus and exemestane in a small number of patients with HR+/HER2- metastatic cancer. Of the 15 patients that were evaluable for response assessment, the ORR was 33\%, the clinical benefit rate at 6 months being $73 \%$. Recently, metastatic breast cancer patients treated with the mTOR inhibitor everolimus, as first line therapy, have been subsequently treated with fulvestrant and palbociclib, resulting in a significantly better progression-free survival (Herrscher et al., 2019). Currently, there are several ongoing trials exploring combination of abemaciclib, ribociclib and palbociclib with agents targeting the PI3K/mTOR pathway, including pan-PI3K inhibitors (copanlisib), alpha-specific PI3K inhibitors (alpelisib, GDC-0077), PI3K/mTOR dual inhibitor (LY3023414), and mTOR inhibitors (everolimus).

It is reasonable to think that in some patients there is the possibility to restore sensitivity to CDK4/6 inhibitors. This could depend on which molecular mechanism is responsible for the resistance. These studies need to be further developed; in fact, they could reveal biomarkers to identify which patients will develop resistance and which, among them, can recover sensitivity to CDK4/6 inhibitors. Indeed, the identification of patients who develop resistance to CDK4/6 inhibitors due to the activation of $\mathrm{PI} 3 \mathrm{~K} / \mathrm{AKT} / \mathrm{mTOR}$ pathway will pave the way to the combination of abemaciclib, ribociclib or palbociclib with everolimus, alpelisib or copanlisib into daily clinical practice. This will represent a major step forward on the road to precision medicine in patients with advanced breast cancer.

\section{CONCLUSION}

The reconstruction of pathways around estrogen receptor (ER), mTOR and cyclin D allowed the comparison of the effects of $\mathrm{CDK} 4 / 6$ and $\mathrm{PI} 3 \mathrm{~K} / \mathrm{AKT} / \mathrm{mTOR}$ inhibitors in metastatic $\mathrm{HR}+$ cancer. Pre-treatment with mTOR pathway inhibitors could prevent or delay the onset of resistance to CDK4/6 inhibitors. Additional studies are needed in order to find the biomarkers that can identify patients who will develop this resistance and in whom the sensitivity to CDK4/6 inhibitors can be restored. Moreover, the combination of anti-mTOR/PI3K/AKT agents with CDK4/6 inhibitors should be further investigated basing on the results obtained in preliminary studies. 


\section{AUTHOR CONTRIBUTIONS}

FP, MS, and AC: conception and design. GS and MC: acquisition of data. GO, MG, and FP: analysis and interpretation of data.

\section{REFERENCES}

Asghar, U., Witkiewicz, A. K., Turner, N. C., and Knudsen, E. S. (2015). The history and future of targeting cyclin-dependent kinases in cancer therapy. Nat. Rev. Drug Discov. 14, 130-146. doi: 10.1038/nrd4504

Bachelot, T., Bourgier, C., Cropet, C., Ray-Coquard, I., Ferrero, J. M., Freyer, G., et al. (2012). Randomized phase II trial of everolimus in combination with tamoxifen in patients with hormone receptor-positive, human epidermal growth factor receptor 2-negative metastatic breast cancer with prior exposure to aromatase inhibitors: a GINECO study. J. Clin. Oncol. 30, 2718-2724. doi: 10.1200/JCO.2011.39.0708

Ballinger, T. J., Meier, J. B., and Jansen, V. M. (2018). Current landscape of targeted therapies for hormone-receptor positive, HER2 negative metastatic breast cancer. Front. Oncol. 8:308. doi: 10.3389/fonc.2018.00308

Bonelli, M. A., Digiacomo, G., Fumarola, C., Alfieri, R., Quaini, F., Falco, A., et al. (2017). Combined inhibition of CDK4/6 and PI3K/AKT/mTOR pathways induces a synergistic anti-tumor effect in malignant pleural mesothelioma cells. Neoplasia 19, 637-648. doi: 10.1016/j.neo.2017.05.003

Bosch, A., Li, Z., Bergamaschi, A., Ellis, H., Toska, E., Prat, A., et al. (2015). PI3K inhibition results in enhanced estrogen receptor function and dependence in hormone receptor-positive breast cancer. Sci. Transl. Med. 7:283ra51. doi: 10.1126/scitranslmed.aaa4442

Campbell, R. A., Bhat-Nakshatri, P., Patel, N. M., Constantinidou, D., Ali, S., and Nakshatri, H. (2001). Phosphatidylinositol 3-kinase/AKT-mediated activation of estrogen receptor alpha: a new model for anti-estrogen resistance. J. Biol. Chem. 276, 9817-9824. doi: 10.1074/jbc.m010840200

Ciruelos Gil, E. M. (2014). Targeting the PI3K/AKT/mTOR pathway in estrogen receptor-positive breast cancer. Cancer Treat. Rev. 40, 862-871. doi: 10.1016/j. ctrv.2014.03.004

Cortes, J., Im, S. A., Holgado, E., Perez-Garcia, J. M., Schmid, P., and ChavezMacgregor, M. (2017). The next era of treatment for hormone receptorpositive, HER2-negative advanced breast cancer: triplet combination-based endocrine therapies. Cancer Treat. Rev. 61, 53-60. doi: 10.1016/j.ctrv.2017. 09.011

Creighton, C. J., Fu, X., Hennessy, B. T., Casa, A. J., Zhang, Y., Gonzalez-Angulo, A. M., et al. (2010). Proteomic and transcriptomic profiling reveals a link between the PI3K pathway and lower estrogen-receptor (ER) levels and activity in ER+ breast cancer. Breast Cancer Res. 12:R40. doi: 10.1186/bcr2594

Cretella, D., Ravelli, A., Fumarola, C., La Monica, S., Digiacomo, G., Cavazzoni, A., et al. (2018). The anti-tumor efficacy of CDK4/6 inhibition is enhanced by the combination with $\mathrm{PI} 3 \mathrm{~K} / \mathrm{AKT} / \mathrm{mTOR}$ inhibitors through impairment of glucose metabolism in TNBC cells. J. Exp. Clin. Cancer Res. 37:72.

De Marchi, T., Foekens, J. A., Umar, A., and Martens, J. W. (2016). Endocrine therapy resistance in estrogen receptor (ER)-positive breast cancer. Drug Discov. Today 21, 1181-1188. doi: 10.1016/j.drudis.2016.05.012

Dhakal, A., Matthews, C. M., Levine, E. G., Salerno, K. E., Zhang, F., Takabe, K., et al. (2018). Efficacy of palbociclib combinations in hormone receptor-positive metastatic breast cancer patients after prior everolimus treatment. Clin. Breast Cancer 18, e1401-e1405. doi: 10.1016/j.clbc.2018.04.015

Di Leo, A., Jerusalem, G., Petruzelka, L., Torres, R., Bondarenko, I. N., Khasanov, R., et al. (2014). Final overall survival: fulvestrant $500 \mathrm{mg}$ vs $250 \mathrm{mg}$ in the randomized CONFIRM trial. J. Natl. Cancer Inst. 106:djt337. doi: 10.1093/jnci/ djt337

Fry, D. W., Harvey, P. J., Keller, P. R., Elliott, W. L., Meade, M., Trachet, E., et al. (2004). Specific inhibition of cyclin-dependent kinase 4/6 by PD 0332991 and associated antitumor activity in human tumor xenografts. Mol. Cancer Ther. 3, 1427-1438.

Giuliano, M., Trivedi, M. V., and Schiff, R. (2013). Bidirectional crosstalk between the estrogen receptor and human epidermal growth factor receptor 2 signaling pathways in breast cancer: molecular basis and clinical implications. Breast Care (Basel) 8, 256-262. doi: 10.1159/000354253
GO and ER: drafting the manuscript. LC, GP, and RM: critical revision of the manuscript for important intellectual content. $\mathrm{AM}$ and NS: technical and material support. $\mathrm{MG}, \mathrm{NB}$, and FP: supervision.

Hamilton, E., and Infante, J. R. (2016). Targeting CDK4/6 in patients with cancer. Cancer Treat. Rev. 45, 129-138. doi: 10.1016/j.ctrv.2016.03.002

Herrera-Abreu, M. T., Palafox, M., Asghar, U., Rivas, M. A., Cutts, R. J., GarciaMurillas, I., et al. (2016). Early adaptation and acquired resistance to CDK4/6 inhibition in estrogen receptor-positive breast cancer. Cancer Res. 76, 23012313. doi: 10.1158/0008-5472.CAN-15-0728

Herrscher, H., Velten, M., Leblanc, J., Kalish-Weindling, M., Fischbach, C., Exinger, D., et al. (2019). Fulvestrant and palbociclib combination in heavily pretreated hormone receptor-positive, HER2-negative metastatic breast cancer patients. Breast Cancer Res. Treat. 179, 371-376. doi: 10.1007/s10549-019-05439-x

Hosford, S. R., and Miller, T. W. (2014). Clinical potential of novel therapeutic targets in breast cancer: CDK4/6, Src, JAK/STAT, PARP, HDAC, and PI3K/AKT/mTOR pathways. Pharmgenomics Pers. Med. 7, 203-215. doi: 10. 2147/PGPM.S52762

Jansen, V. M., Bhola, N. E., Bauer, J. A., Formisano, L., Lee, K. M., Hutchinson, K. E., et al. (2017). Kinome-wide RNA interference screen reveals a role for PDK1 in acquired resistance to CDK4/6 inhibition in ER-positive breast cancer. Cancer Res. 77, 2488-2499. doi: 10.1158/0008-5472.CAN-16-2653

Kim, L. C., Cook, R. S., and Chen, J. (2017). mTORC1 and mTORC2 in cancer and the tumor microenvironment. Oncogene 36, 2191-2201. doi: 10.1038/onc. 2016.363

Kim, S., Loo, A., Chopra, R., Caponigro, G., Huang, A., Vora, S., et al. (2014). Abstract PR02: LEE011: an orally bioavailable, selective small molecule inhibitor of CDK4/6- Reactivating Rb in cancer. Mol. Cancer Ther. 12:R02.

Laderian, B., and Fojo, T. (2017). CDK4/6 Inhibition as a therapeutic strategy in breast cancer: palbociclib, ribociclib, and abemaciclib. Semin. Oncol. 44, 395-403. doi: 10.1053/j.seminoncol.2018.03.006

Li, Z., Razavi, P., Li, Q., Toy, W., Liu, B., Ping, C., et al. (2018). Loss of the FAT1 tumor suppressor promotes resistance to CDK4/6 inhibitors via the hippo pathway. Cancer Cell 34, 893-905.e8. doi: 10.1016/j.ccell.2018.11.006

Michaloglou, C., Crafter, C., Siersbaek, R., Delpuech, O., Curwen, J. O., Carnevalli, L. S., et al. (2018). Combined inhibition of mTOR and CDK4/6 is required for optimal blockade of E2F function and long-term growth inhibition in estrogen receptor-positive breast cancer. Mol. Cancer Ther. 17, 908-920. doi: 10.1158/ 1535-7163.MCT-17-0537

Milla-Santos, A., Milla, L., Portella, J., Rallo, L., Pons, M., Rodes, E., et al. (2003). Anastrozole versus tamoxifen as first-line therapy in postmenopausal patients with hormone-dependent advanced breast cancer: a prospective, randomized, phase III study. Am. J. Clin. Oncol. 26, 317-322. doi: 10.1097/ 01.coc.0000047126.10522.f9

Miller, T. W., Balko, J. M., and Arteaga, C. L. (2011). Phosphatidylinositol 3-kinase and antiestrogen resistance in breast cancer. J. Clin. Oncol. 29, 4452-4461. doi: 10.1200/JCO.2010.34.4879

Oliveira, M., Chavez-MacGregor, M., Modi, S., Campone, M., Ma, B., Dirix, L., et al. (2016). "Adding ribociclib (LEE011) to everolimus and exemestane in estrogen receptor-positive $(\mathrm{ER}+)$ /human epidermal growth factor receptor 2-negative (HER2-) advanced breast cancer (ABC): feasibility and possible benefits," in Proceedings of the 2nd World Congress on Controversies in Breast Cancer, (Barcelona: COBRCA).

Olmez, I., Brenneman, B., Xiao, A., Serbulea, V., Benamar, M., Zhang, Y., et al. (2017). Combined CDK4/6 and mTOR inhibition is synergistic against glioblastoma via multiple mechanisms. Clin. Cancer Res. 23, 6958-6968. doi: 10.1158/1078-0432.CCR-17-0803

Osborne, C. K., and Schiff, R. (2011). Mechanisms of endocrine resistance in breast cancer. Annu. Rev. Med. 62, 233-247. doi: 10.1146/annurev-med-070909182917

Pandey, K., An, H. J., Kim, S. K., Lee, S. A., Kim, S., Lim, S. M., et al. (2019). Molecular mechanisms of resistance to CDK4/6 inhibitors in breast cancer: a review. Int. J. Cancer 145, 1179-1188. doi: 10.1002/ijc.32020

Platet, N., Cathiard, A. M., Gleizes, M., and Garcia, M. (2004). Estrogens and their receptors in breast cancer progression: a dual role in cancer proliferation and 
invasion. Crit. Rev. Oncol. Hematol. 51, 55-67. doi: 10.1016/j.critrevonc.2004. 02.001

Rosenwald, I. B., Kaspar, R., Rousseau, D., Gehrke, L., Leboulch, P., Chen, J. J., et al. (1995). Eukaryotic translation initiation factor $4 \mathrm{E}$ regulates expression of cyclin D1 at transcriptional and post-transcriptional levels. J. Biol. Chem. 270, 21176-21180. doi: 10.1074/jbc.270.36.21176

Toogood, P. L., Harvey, P. J., Repine, J. T., Sheehan, D. J., Vanderwel, S. N., Zhou, H., et al. (2005). Discovery of a potent and selective inhibitor of cyclindependent kinase 4/6. J. Med. Chem. 48, 2388-2406.

Yamamoto, T., Kanaya, N., Somlo, G., and Chen, S. (2019). Synergistic anticancer activity of CDK4/6 inhibitor palbociclib and dual mTOR kinase inhibitor MLN0128 in pRb-expressing ER-negative breast cancer. Breast Cancer Res. Treat. 174, 615-625. doi: 10.1007/s10549-018-05104-9

Yamnik, R. L., Digilova, A., Davis, D. C., Brodt, Z. N., Murphy, C. J., and Holz, M. K. (2009). S6 kinase 1 regulates estrogen receptor alpha in control of breast cancer cell proliferation. J. Biol. Chem. 284, 6361-6369. doi: 10.1074/jbc.M807532200

Yardley, D. A., Noguchi, S., Pritchard, K. I., Burris, H. A. III, Baselga, J., Gnant, M., et al. (2013). Everolimus plus exemestane in postmenopausal patients with
$\mathrm{HR}(+)$ breast cancer: BOLERO-2 final progression-free survival analysis. $A d v$. Ther. 30, 870-884. doi: 10.1007/s12325-013-0060-1

Zhang, J., Xu, K., Liu, P., Geng, Y., Wang, B., Gan, W., et al. (2016). Inhibition of $\mathrm{Rb}$ phosphorylation leads to mTORC2-mediated activation of Akt. Mol. Cell 62, 929-942. doi: 10.1016/j.molcel.2016.04.023

Conflict of Interest: The authors declare that the research was conducted in the absence of any commercial or financial relationships that could be construed as a potential conflict of interest.

Copyright (C) 2020 Occhipinti, Romagnoli, Santoni, Cimadamore, Sorgentoni, Cecati, Giulietti, Battelli, Maccioni, Storti, Cheng, Principato, Montironi and Piva. This is an open-access article distributed under the terms of the Creative Commons Attribution License (CC BY). The use, distribution or reproduction in other forums is permitted, provided the original author(s) and the copyright owner(s) are credited and that the original publication in this journal is cited, in accordance with accepted academic practice. No use, distribution or reproduction is permitted which does not comply with these terms. 